there be heritability for the male trait which improves survival and attracts females. This finding is reminiscent of Partridge's ${ }^{6}$, showing that male Drosophila that succeed in mating competition also tend to produce larvae with high survivorship, and of Watt et al. ${ }^{7}$, showing that certain genotypes in male Colias butterflies receive both a mating and a survival advantage.

Supporters of the nonadaptive view, however, will be quick to point out that showing that mate choice in pheasants is consistent with the good-genes view falls short of showing that the good-genes mechanism is actually at work. First, the observation that females mated to longspurred males fledge more offspring has not been tied to a genetic effect from the fathers. It is possible, for example, that these females are laying more eggs or are giving them better care as a consequence of mating with these males. This possibility is suggested by Burley's ${ }^{8}$ work on zebra finches showing that mating with a preferred male causes females to expend more parental care than they would otherwise.
Second, and much more difficult, is the point that these data focus on the male trait, while the controversy revolves around the evolution of the female preference. Whereas the pheasant data are consistent with the good-genes hypothesis, they are also consistent with alternatives. A definitive resolution of the controversy may have to wait for a system in which the evolutionary forces and inheritance of female preferences themselves can be studied. Until that goal is realized, we may be able to show how some males get a free lunch, but not why females choose to sit at their table.

1. Von Schantz, T. et al. Nature 337, 166-169 (1989).

2. Darwin, C. On the Origin of Species (Murray, London, 1859).

3. Darwin, C. The Descent of Man, and Selection in Relation to Sex (Murray, London, 1871).

4. Kirkpatrick, M. A. Rev. Ecol. Syst. 18, 43-70 (1987).

5. Bradbury, J.W. \& Andersson, M.B. Sexual Selection: Testing the Alternatives (Wiley, Chichester, 1987).

6. Partridge, L. Nature 283, 290-291 (1980)

7. Watt, W.B. et al. Science 233,1187-1190 (1986).

8. Burley, N. Am. Nat. 132, 611-628 (1988).

Mark Kirkpatrick is in the Department of Zoology, University of Texas, Austin, Texas 78712, USA.

\title{
Zeolites
}

\section{Multidimensional large pores}

\section{Mark E. Davis}

ZEOLITES and zeolite-like materials comprise a broad range of microporous, crystalline solids whose main uses are as adsorbents, catalysts and ion exchangers. These solids have tunable properties such as pore size, shape and capacity, and lead one to believe that designer catalysts are possible through the molecular engineering of zeolite structures (frameworks). Brunner and Meier on page 146 of this issue $^{1}$ report findings which provide insights for future synthesis directions which could lead to new zeolites with properties that are not currently available.

The basic building blocks of zeolites are tetrahedra of $\mathrm{SiO}_{4}$ or $\mathrm{AlO}_{4}$, which connect to form complex networks of one-, two- or three-dimensional molecular-sized tunnels. The pores can be used to sort molecules, and their surfaces to catalyse reactions.

Brunner and Meier show that there is a correlation between the minimum framework density (FD) defined as the number of tetrahedral atoms (T-atoms) per cubic nanometre and the smallest ring size in the framework. Clearly, the FD is related to the void volume of the crystal: as the FD decreases, the void volume or capacity for adsorption increases. The minimum known FD is 12.5 which corresponds to the void occupying just over half the crystal volume. Materials with an FD more than 12 contain rings consisting of 4 or more $\mathrm{T}$-atoms (4-membered rings). The exception is lovdarite ${ }^{2}$ which possesses 3-membered rings, some of which have Tatoms shared by 4 -membered rings. Thus, Brunner and Meier designate it as 3+.

If the correlation between the minimum FD and ring size is correct, then zeolites with 3-membered rings must be synthesized to achieve materials with a higher capacity. Meier ${ }^{3}$ has offered several hypothetical structures which are based upon 3 -membered ring building units with FDs in the range of $9-10$. These very open networks are quite fascinating and lead one to speculate upon uses of such materials if they could be synthesized. One particularly important application could be the effective storage of methane.

Numerous hypothetical frameworks are

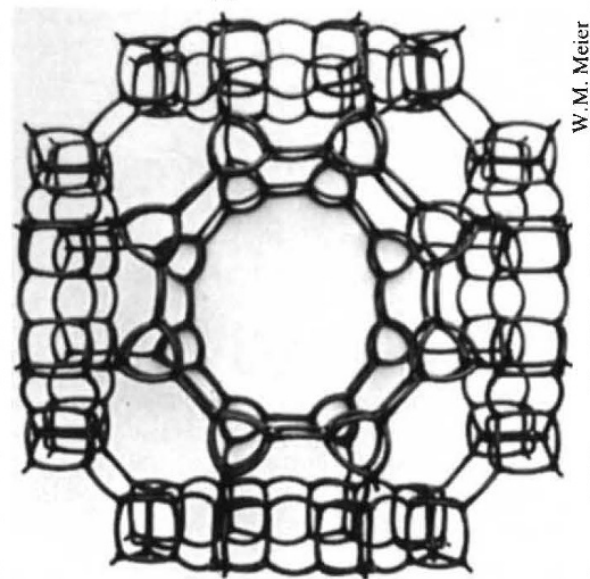

Hypothetical framework with large voids and three-dimensional extra-large pores. given in the open literature (besides those in confidential documents) which stimulate the synthetic chemist. Until very recently, for example, the largest ring in zeolites or molecular sieves contained $12 \mathrm{~T}$-atoms. But there are hypothetical frameworks with 18- and 24-membered rings ${ }^{4,5}$. Synthetic strategies developed from these hypothesized ring structures led us to synthesize the first extra-large pore molecular sieve which contained 18-membered rings ${ }^{6}$.

One of the new frontiers in zeolite science is the synthesis of extra-large pore materials. The availability of zeolites with pores 1-2 nanometres across would open new catalytic chemistry in the petrochemical and life-sciences fields. The predictions of Brunner and Meier are particularly germane to this goal. The one known 18-membered ring structure has an FD of 14.2 , and the hypothetical 24-membered ring structures have FDs below 12 (ref. 4). All of these frameworks contain 4-membered rings. Also, these networks possess one-dimensional channels which are circumscribed by the extra-large rings.

Meier $^{3}$ speculates that all extra-large ring structures based upon 4-membered rings will have one-dimensional channel systems. This is because hypothetical frameworks with multidimensional extralarge channels yield FDs below 12. Onedimensional channel systems in existing zeolites do not produce particularly good catalysts: because reactant and product molecules have one way into and one way out of the structure, the molecule flow is extremely congested which leads to poor catalytic activity. On the other hand, if the structure contains pore systems with greater dimensionality, then the molecular traffic can be routed to enhance movement of reactants to the catalytic sites and removal of the products. Meier ${ }^{3}$ claims that a 16-membered ring framework can be derived using 3-membered ring units with $\mathrm{FD}=9.3$ (see figure). Thus, extra-large pore structures with multidimensional channel systems may be feasible.

The existence of lovdarite lends hope that frameworks based upon 3-membered rings can be synthesized. But because lovdarite contains beryllium, a highly toxic component, practical application of the concepts of Brunner and Meier rest on discovery of synthetic routes to 3-membered rings with elements other than beryllium.

1. Brunner, G. O. \& Meier, W. M. Nature 337. 146-147 (1989).

Merlino, S. Acta Cryst. A37. C189 (1981)

3eier, W. M. New Developments in Zeolite Science and Technology (eds Murakami, X.. Iijima, A. \& Ward. J. W.) 13-22 (Elsevier. Amsterdam. 1986).

Smith, J. V. \& Dytrych. W. J. Nature 309.607-608 (1984)

. Barrer. R. M. \& Villiger, H. Z. Kristallogr. 128, 352-370 (1963).

6. Davis. M.E. et al. Nature 331, 698-699 (1988)

Mark E. Davis is in the Department of Chemical Engineering, Virginia Polytechnic and State University, Blacksburg, Virginia 24061, USA. 